\section{Study on irradiated D-mannose isolated from cranberry}

\begin{abstract}
The stable EPR signal produced by ionizing radiation in crystalline D-mannose $\left(\mathrm{C}_{6} \mathrm{H}_{12} \mathrm{O}_{6}\right)$ and separated from cranberries (Vaccinium oxycoccus) was studied. The isothermal heating of irradiated sample at $95^{\circ} \mathrm{C}$ for 10 minutes (melting point of D-mannose is $132^{\circ} \mathrm{C}$ ) resulted in the modification and simplification of the EPR signal involved. The isotropic quartet has been recognized in the EPR signal of heat-treated sample. Molecular structure of the isotropic quartet identified in the complex EPR signal of D-mannose crystallite is proposed.
\end{abstract}

Keywords: crystallite $\bullet$ electron paramagnetic resonance $(\mathrm{EPR}) \bullet$ irradiation $\bullet$ mannose

G. P. Guzik ${ }^{\bowtie}$, J. Michalik

Institute of Nuclear Chemistry and Technology

16 Dorodna St., 03-195 Warsaw, Poland

E-mail: g.guzik@ichtj.waw.pl

W. Stachowicz

Expert on Food Irradiation, emeritus

Warsaw, Poland

Received: 16 November 2018

Accepted: 16 September 2019

\section{Introduction}

The stable EPR signal evoked in dried fruits by the action of ionizing radiation has been proposed as a marker of radiation treatment [1] and as such was tested and positively evaluated in interlaboratory exercises on international level $[1,2]$. Analytical procedure for the detection of radiation treatment in fruits based on the EPR spectroscopy has been approved and issued by CEN as European standard EN 13708. The latter is recommended for the application in the food control laboratories as a tool for the detection of irradiation in food containing crystalline sugars [1]. The method is currently applied worldwide for the control of dried fruits, if irradiated. However, sugar and water contents in dried fruits vary markedly [3, 4], which results in consequence higher or lower concentration of crystalline sugar domains followed by higher and lower intensity of the EPR signal involved. The lack of the EPR signal proves the absence of the controlled fruit of sugars in the crystalline form. The appearance and/or the lack of crystalline sugars in dried fruits depends also on the specificity of the product undergoing investigation. No crystalline sugar has been found in dried plum and dried apricot for example.

There has not been found in the literature any information on the identity of long-lived radical trapped in irradiated D-mannose giving rise in EPR to multiline spectrum (EN 13708 standard). It has not been proven yet whether mannose radiation born EPR signal is suitable to be employed as the indicator of radiation treatment in mannose-containing fruits. 
In our earlier EPR studies on crystalline sugars separated from fruits, we focused our efforts on the separation of isotropic EPR spectra responsible for the dominating lines in the complex EPR spectra of irradiated fructose [5], glucose [6] and sorbose [7]. The study on the stable EPR spectrum of X irradiated single crystals of L-sorbose [8] is the only publication found in the literature related to the subject. The molecular structures of radicals involved have been proposed too. Possibly, it was achieved by applying of an original method of the separation of isotropic radical spectra from initial multiline EPR signals by the comparison and/or subtraction of the EPR spectra recorded with unheated samples and those heated to the temperatures slightly below the temperature of the melting points of sugars involved [5].

Cranberry is a fruit commercially harvested and distributed through world food markets particularly in the United States, Canada (Vaccinium macrocarpon) and Great Britain (Vaccinium oxycoccus). It is traditionally believed that cranberry is helpful in maintaining the good functioning of urinary system. However, the recent medical publications do not confirm a positive action of cranberry in preventing the urinary system against infections $[9,10]$. Raw cranberries contain $12.2 \mathrm{~g} / 100 \mathrm{~g}$ of carbohydrates including sugars $(4.04 \mathrm{~g} / 100 \mathrm{~g})$, dietary fibre $(4.6 \mathrm{~g} / 100 \mathrm{~g})$, vitamin $\mathrm{C}$ and the essential dietary minerals [4]. As fresh cranberry is rather hard and bitter in taste, most of the harvested cranberry (up to $95 \%$ ) is processed to obtain sweetened cranberry fruit, sauce or juice $[11,12]$. The dominating sugar identified in cranberry is mannose (up to $3 \%$ of the fruit) while the other sugars appear in much smaller concentration.

Synthetic mannose is obtained by the oxidation of mannitol, which is the product isolated from the manna a substance taken from manna tree (Fraxinus ornus) and some other trees and shrubs. Mannose as a C-2 epimer of glucose can be also produced from glucose by chemical transformation proceeding by the inversion of C-2 chiral centre of glucose. The change in molecular configuration of glucose and mannose hexoses occurs in some of the biochemical processes too.

It has to be announced that mannose plays some role in human metabolism taking part in the glycosylation of proteins [13]. While most of the mannose participating in glycosylation process is believed to be derived from glucose, it was also observed that in hepatoma cell colony (cancerous cells of the liver) the glycoprotein biosynthesis comes from the extracellular mannose but not from glucose indeed [14]. Many of the glycoproteins produced in the liver are present in the bloodstream, so the dietary mannose is spread throughout the body $[15,16]$.
Mannose structurally appears in two different size rings: pyranose (six-link ring) and furanose (five-link ring). Pyranose form is dominant in 99.8\% in plants (i.e. in almost all commercial fruits) and mushrooms (tea tree mushroom). Each of the cited ring forms of mannose appears either in alpha or beta configuration at the anomeric position. Alpha-D-mannopyranose structure appears in 67\% in soft fruits containing as dominating component water) as, for example, in fresh cranberry [17].

\section{Materials and methods}

Fresh cranberries were purchased in the market and subsequently washed with water. All single berries were cut into smaller pieces and crushed to a pulp. The pulp was then dried for 48 hours in $40^{\circ} \mathrm{C}$ in the laboratory dryer under the flow of dry air. The above preparation procedure simulated the steps of commercial production of dried cranberries cane sugar free. A part of the dried cranberry pulp was taken for the EPR study on a possible application of the EPR method for the detection of radiation treatment of cranberry (see the next Section). The remaining volume of the pulp undergoes procedure to extract sugars. The pulp was diluted with a suitable volume of demineralized water and stirred. The obtained pulp suspension was kept quiet for several hours while clear solution from over the organic residue was carefully decanted. The final step was crystallization of sugar crystallites from water solution at room temperature. Clear solution is poured to the opened Petri dishes and kept motionless under static dust-free condition for at least 7 weeks in open air. Slow crystallization occurred gradually. At a suitable moment, the crystalline sediment (crystallite) was carefully taken off from saturated solution and dried. The identity of isolated crystallites has been proven by refraction measurements with the use of Rudolf 3357 refraction meter. The refraction coefficient $\mu$ of the isolated crystallite $(\mu=1.34596)$ was found very close to that of pure crystalline mannose $(\mu=1.34604)$ [18]. It is a cogent argument for the identity of the extracted cranberry crystallite with crystalline mannose (see Table 1).

The Sigma-Aldrich crystalline mannose, $98 \%$ re-crystallized in water, was used as referent material in the following EPR study on cranberry crystallites.

Dried cranberry pulp and D-mannose crystallite extracted from cranberry were irradiated in ${ }^{60} \mathrm{Co}$ gamma source (Gamma Chamber 5000) with the doses of $0.5,1,2$, and $3 \mathrm{kGy}$, that is, within the range of doses recommended in normative documents for dried fruits [19-21]. The lowest dose of $0.5 \mathrm{kGy}$ lies

Table 1. Specificity of crystalline mannose isolated from dried cranberries

Form and size of mannose crystals

Small aggregates composed of orthorhombic crystals ca. $<0.01 \mathrm{~mm}$ long

\begin{tabular}{lcc}
\hline $\begin{array}{l}\text { Solvents used for slow room temperature } \\
\text { crystallization }\end{array}$ & $\begin{array}{c}\text { Distilled and demineralized } \\
\text { water }\end{array}$ & $\begin{array}{c}\text { Ethanol 98\% of chemical } \\
\text { purity }\end{array}$ \\
Refraction coefficient $(\mu) 20^{\circ} \mathrm{C}$ & $1.34596 \pm 0.00004$ & $1.34617 \pm 0.00013$ \\
Refraction coefficient $(\mu)$ of mannose, Ref. $[18]$ & $1.34604 \pm 0.00001$ & $1.34604 \pm 0.00001$ \\
\hline
\end{tabular}


below this range and is added to prove the detection sensitivity of the EPR detection method used.

The EPR measurements were executed 1 day (at least 24 hours) after radiation treatment to avoid the recording of the EPR signals that derived from short-lived radicals overlapping the investigated EPR signals. This kind of short-lived EPR is observed quite often immediately after radiation treatment in food but decays within a few hours. The EPR operational parameters adapted were taken from EN 13708 European standard [1]. The samples of dried cranberry pulp and of mannose crystallite weighing $100 \pm 2 \mathrm{mg}$ were placed in the EPR signal-free glass ampoules $4 \mathrm{~mm}$ in diameter and measured in X band with Bruker EPR spectrometer model EMX plus equipped with Xenon software program.

\section{Results and discussion}

\section{EPR spectra of dried cranberry treated with radiation}

The relationship between the EPR signal intensities (integrated EPR signal areas) recorded with the samples of dried cranberry pulp treated and the doses of ionizing radiation used is shown in Fig. 1. Linear increase in the EPR signal intensity with the increasing dose of ionizing radiation is typical for radiation originated radicals. The shape of the EPR signals recorded proves also its suitability to be a specific marker for the detection of irradiation in dried cranberry.

The multiline, broad EPR signal recorded with dried cranberry pulp irradiated with $3 \mathrm{kGy}$ is shown in the upper part of Fig. 1.

The EPR examination of not irradiated cranberry pulp did not prove any EPR signal, although slight departure from the linearity of the baseline is stated resulting in a small shift of the intersection of the straight line relationship as seen in the graph (Fig. 1). The EPR signal recorded with the sample irradiated with $0.5 \mathrm{kGy}$ was weak but sill detectable in EPR (signal-to-noise ratio $>4$ ).

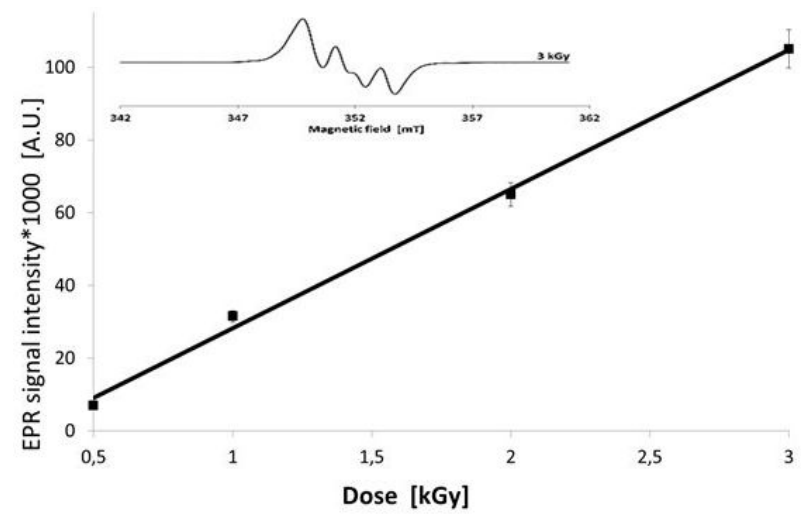

Fig. 1. The relationship between the EPR signal intensity and the dose of radiation in dried cranberry pulp. The EPR signals were measured 1 day after radiation treatment. The doses of ${ }^{60} \mathrm{C}$ radiation applied were as follows: $0.5,1,2$, and $3 \mathrm{kGy}$, respectively. Room temperature measurements were made. The weight of samples measured was $100 \pm$ $2 \mathrm{mg}$. In the upper window, the EPR signal recorded with cranberry pulp irradiated with $3 \mathrm{kGy}$ is shown.

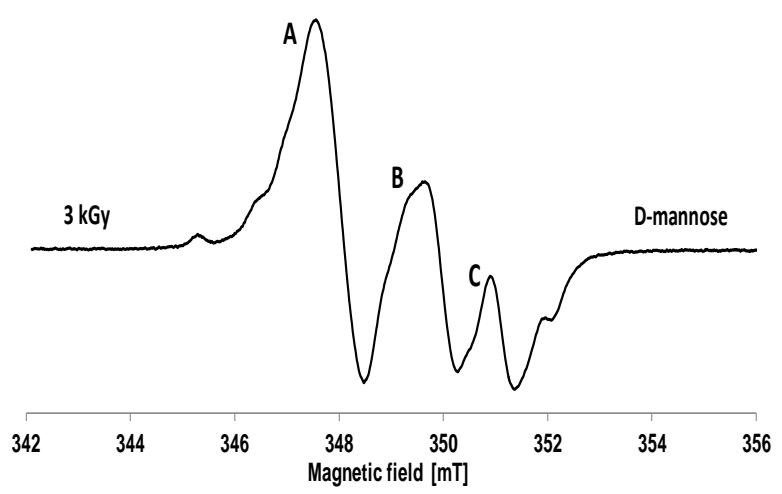

Fig. 2. EPR signal of the crystallite extracted from dried cranberry and irradiated with the dose of $3 \mathrm{kGy}$ of ${ }^{60} \mathrm{Co}$ gamma rays. The record was taken 1 day after radiation treatment at room temperature. The weight of the crystallite sample was $100 \pm 2 \mathrm{mg}$.

\section{EPR spectra of D-mannose crystallites isolated from water solutions extracted from cranberry}

The EPR signals obtained with irradiated referent sample used (Sigma-Aldrich re-crystallized D-mannose) were identical with those taken with irradiated crystallite matrix separated from cranberry. This is an additional argument confirming the earlier presupposition based on the comparison of refraction coefficients (Table 1) that separated crystallite is composed of D-mannose only. In Fig. 2, the EPR signal obtained with D-mannose crystallite matrix is shown. The sample undergoing the measurement was kept at room temperature. The dose of gamma radiation applied was $3 \mathrm{kGy}$.

The signal is relatively broad with three dominating lines (A, B and C). In comparison with the EPR signals of irradiated glucose or fructose recorded in earlier studies $[5,6]$, it is simpler and less asymmetric. However, no spectral lines that could be assigned to isotropic spectrum of a single radical could be distinguished. The comparison between the EPR signals of dried cranberry pulp and those of crystalline D-mannose (referent sample of D-mannose of Sigma-Aldrich re-crystallized) both irradiated with $3 \mathrm{kGy}$ of ${ }^{60} \mathrm{Co}$ gamma rays and normalized is shown in Fig. 3.

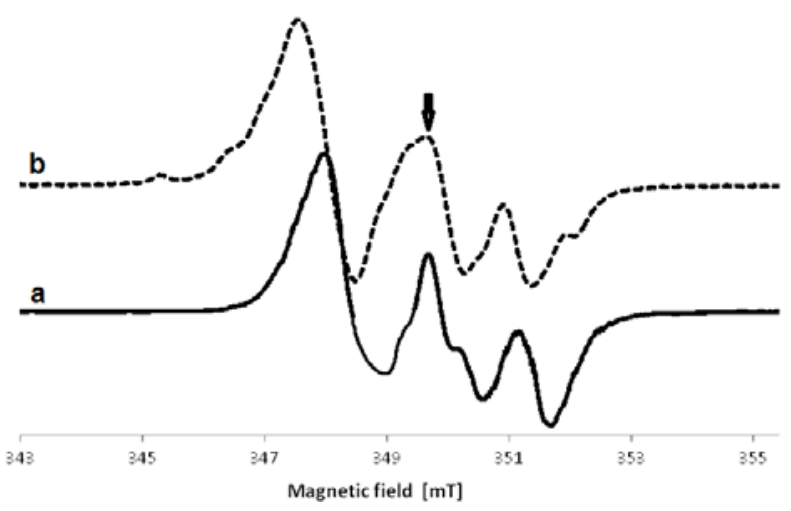

Fig. 3. EPR signals taken 1 day after the irradiation at room temperature of dried cranberry (a) and of re-crystallized Sigma-Aldrich D-mannose (b) both irradiated with $3 \mathrm{kGy}$ of ${ }^{60} \mathrm{Co}$ gamma rays. The spectra are normalized to the same EPR signal intensity. 


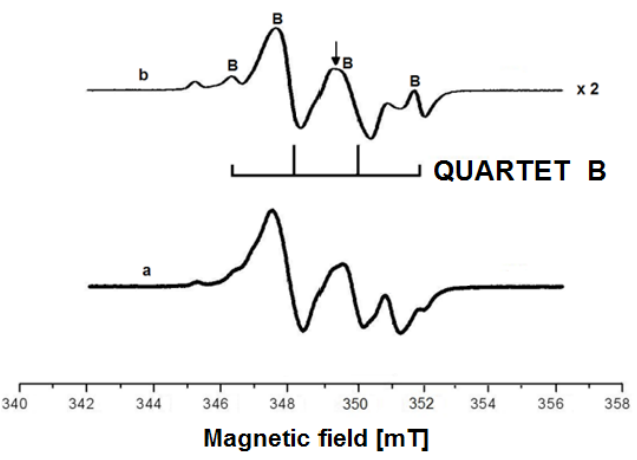

Fig. 4. EPR signal of crystalline D-mannose obtained from dried cranberry irradiated with the dose of $4 \mathrm{kGy}$ of gamma rays and heated after radiation treatment for 10 minutes at $95^{\circ} \mathrm{C}$ (the upper spectrum $\mathrm{b}$ ). The isotropic quartet of $\mathrm{A}_{\text {iso }}=1.8 \pm 0.2 \mathrm{mT}$, denoted $\mathrm{B}-\mathrm{B}-\mathrm{B}-\mathrm{B}$, is distinguished. The lower spectrum (a) was taken with the same sample but before heating.

Both EPR signals are obviously different, although it is possible to distinguish three dominating lines in them. The above observation confirms the earlier supposition that D-mannose is the only component of the crystallite matrix separated from cranberry pulp.

\section{EPR spectra of D-mannose crystallites irradiated and heated at $95^{\circ} \mathrm{C}$}

In Fig. 4, the EPR signal of irradiated crystallite of D-mannose is shown, extracted from dried cranberry pulp and heated at $95^{\circ} \mathrm{C}$ for 10 minutes (upper spectrum) compared with the signal of the same but unheated crystallite matrix (lower spectrum). In both spectra, the main spectral lines are distinguished but of relative intensities obviously changed. The EPR signal of heated D-mannose sample becomes less asymmetric as compared with that recorded with unheated sample. It is probably the effect of the decreasing anisotropy of the primary signal during the heating. In the temperature-modified signal, it is possible to distinguish the isotropic quartet designated B-B-B-B as shown in the upper graph in Fig. 4. The splitting factor of the identified quartet is $\mathrm{A}_{\text {iso }}=1.8 \pm 0.2 \mathrm{mT}$ while its $\mathrm{g}_{\mathrm{o}}$ value equals to 2.004. The computer subtraction of the normalized spectra of heated sample from that of primary one, the procedure successful in the earlier studies [5, 6], did not deliver, in contrast to the latter, the EPR signal which could be assigned to the spectrum of less thermally stable radical decaying under heating. It could be concluded, therefore, that radical giving rise to the isotropic quartet identified in EPR is a dominating and perhaps the only one relatively stable paramagnetic entity produced by radiation in irradiated D-mannose.

\section{The radical model of the mannose molecule based on EPR experimental data}

Discrete Fourier theory (DFT) simulation procedure including relevant quantum-mechanical calculation and fittings based on the Gaussian program 03W (demonstration version) were employed to get the most probable molecular structure of radical giving rise to a quartet distinguished in the EPR signal recorded with the heated D-mannose sample.

It has been deduced that among several structural models considered the best fitting was attained with that representing the occurrence of unpaired electron centred at C-5 carbon atom of pyranose ring. Localized at C-5 bond of mannose radical carbon atom skeleton, unpaired electron interacts equivalently with three nuclei of hydrogen atoms: two bonded with C- 6 carbon atom of pyranose ring and one bonded with C-4 atom of $-\mathrm{H}_{2} \mathrm{OH}$ group (Fig. 5).

Spectroscopic slitting factor $\mathrm{A}_{\text {iso }}$ of the EPR quartet derived from $\mathrm{D}$-mannose radical obtained experimentally is $\mathrm{A}_{\text {iso }}=1.8 \pm 0.2 \mathrm{mT}$. The $\mathrm{g}_{\mathrm{o}}$ value obtained from spectroscopic analysis of experimental signal of the quartet was 2.004. Similar numbers are obtained with most of the aliphatic radicals of different molecular structure by their EPR examination [22]. Depending on which carbon atom of pyranose ring the unpaired electron is centred, its interaction with hydrogen atoms of the molecule is different. The fittings done with the unpaired electron centred at carbon atoms of pyranose ring different from that of C-5 did not deliver as satisfactory result as that obtained with the structure presented in Fig. 5.

\section{Conclusions}

The EPR results obtained and discussed through the study on the irradiated crystalline D-mannose extracted from dried cranberry delivered new and valuable experimental data, confirming the usefulness of the method of programmed heating as a tool suitable for the EPR investigation of radicals evoked by ionizing radiation in radiation-treated sugars and sugar-containing fruits $[5,6]$. The treatment is not followed by softening of the matrix. It does not mean, however, that the crystalline lattice of heated D-mannose remains unchanged. The results show conclusively that molecular matrix of mannose crystals undergo relaxation under such condition followed by 'smoothing' of their structure reflected in the decreased anisotropy of the EPR signals involved in which the active role play also trapped radicals produced by radiation. Part of these electrons is less strongly bonded with the hosting crystalline lattice

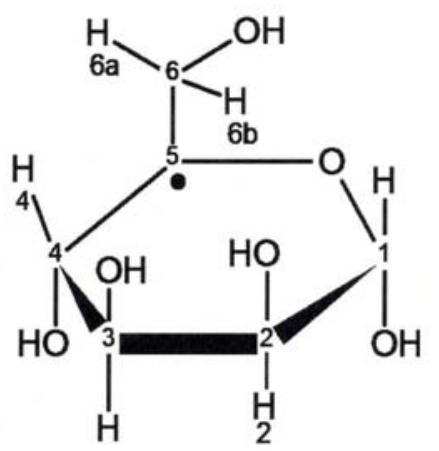

Fig. 5. The probable structure of D-mannose born radical giving rise in EPR to the isotropic quartet B-B-B-B as seen in Fig. 4 (spectrum b). 
and become freed by heating. This is reflected in the EPR records which become less anisotropic and less complex as compared with the primary ones recorded at room temperature. In addition, it was possible to identify for the first time the EPR lines belonging to the isotropic spectra of single radicals responsible for a complex multicomponent EPR signal recorded with irradiated fruits and sugars, as demonstrated in the present and in the earlier studies [5, 6].

Although European standard EN 13708 on the detection of irradiated food containing crystalline sugars is addressed to all kinds of fruits, there are not much information available on the successful detection of radiation treatment in fruits containing D-mannose. Typically, the concentration of mannose in fruit like cranberry is much lower than that in sweet fruits containing fructose and/or glucose. That is why a part of present study was focused on testing the sensitivity of standardized method if applied to the control of mannose-containing fruit. The linear increase in the stable EPR signal intensity in dried cranberry with the increasing dose radiation as well as the detection of radiation treatment of this fruit with the dose of $0.5 \mathrm{kGy}$ approve the applicability of the EPR method as suitable for the routine control of D-mannose-containing fruits if irradiated.

\section{ORCID}

Grzegorz P. Guzik (D) http://orcid.org/0000-0001-9946-3133 Jacek Michalik (D) http://orcid.org/0000-0001-6609-1337

\section{References}

1. European Committee for Standardization (CEN). (2003). European Standard: Foodstuffs - Detection of irradiated food containing crystalline sugar by ESR spectroscopy. EN 13708:2003. (ICS 67.050). Brussels.

2. European Committee for Standardization (CEN). (2001). European Standard: Foodstuffs - Detection of irradiated food containing cellulose by ESR spectroscopy. EN 1787:2001. (ICS 67.050). Brussels.

3. Poradnik fizykochemiczny (2nd ed.). (1974). Warszawa: Wydawnictwa Naukowo-Techniczne WNT.

4. USDA National Nutrient Database for Standard Reference. (2019). Slides almonds and cranberries. Available from Agricultural Research Service, https:// fdc.nal.usda.gov/fdc-app.html\#/food-details/514236/ nutrients.

5. Guzik, G. P., Stachowicz, W., \& Michalik, J. (2008). Study on stable radicals produced by ionising radiation in dried fruits and related sugars by electron paramagnetic resonance spectrometry and photostimulated luminescence method - I. D-fructose. Nukleonika, 53(Suppl. 2), S89-S94.

6. Guzik, G. P., \& Stachowicz, W. (2013). Study on radicals giving rise to multicomponent EMR spectra in dried fruits exposed to ionizing radiation II. D-Glucose. Nukleonika, 58(3), 425-428.

7. Guzik, G. P., \& Stachowicz, W. (2016). Study on radiation-induced radicals giving rise to stable EPR signal suitable for the detection of irradiation in
L-sorbose-containing fruits. Nukleonika, 61(4), 461-465. doi: 10.1515/nuka-2016-0075.

8. Vanhaelewyn, G., Jansen, B., Callens, F. J., \& Sagstuen, E. (2004). ENDOR - Assisted study of the stable EPR spectrum of X-irradiated $\alpha$-L-Sorbose single crystals: MLCFA and simulation decomposition analyses. Radiat. Res., 162, 96-104.

9. Jepson, R. G., Williams, G., \& Craig, J. C. (2013). Cranberries for preventing urinary tract infections. Sao Paulo Med. J., 131(5), 363. DOI: 10.1590/15163180.2013.1315T1

10. European Food Safety Authority. (2014). Scientific opinion on the substantiation of a health claim related to CranMax ${ }^{\circledR}$ and reduction of the risk of urinary tract infection by inhibiting the adhesion of certain bacteria in the urinary tract pursuant to Article 14 of Regulation (EC) No 1924/20061. EFSA Journal, 12(5), 3657.

11. Zeldes, B., \& Leah, A. (2009, November 25). Eat this. Cranberries more than a thanksgiving condiment. Dining Chicago. Chicago's Restaurant \& Entertainment Guide, Inc. Food and Life Periodic of Chicago Restaurants.

12. Eck, P. (1990). The American cranberry. New Brunswick: Rutgers University Press.

13. Freeze, H. H., \& Sharma, V. (2010). Metabolic manipulation of glycosylation disorders in humans and animal models. Semin. Cell Dev. Biol., 21(6), 655-662.

14. Alton, G., Hasilik, M., Niehues, R., Panneerselvam, K., Etchison, J. R., Fana, F., \& Freeze, H. H. (1998). Direct utilization of mannose for mammalian glycoprotein biosynthesis. Glycobiology, 8(3), 285-295.

15. Davis, J. A., \& Freeze, H. H. (2001). Studies of mannose metabolism and effects of long-term mannose ingestion in the mouse. Biochim. Biophys. Acta, 1528(2/3), 116-126.

16. Vlahopoulos, S., Gritzapis, A. D., Perez, S. A., Cacoullos, N., Papamichail, M., \& Baxevanis, C. N. (2009). Mannose addition by yeast Pichia Pastoris on recombinant HER-2 protein inhibits recognition by the monoclonal antibody herceptin. Vaccine, 27(34), 4704-4708.

17. Collins P. M. (Ed.). (2005). Dictionary of carbohydrates (2nd ed.). Boca Raton: CRC Press.

18. The President of the Office of the Registration of Curative Products, Medical Goods and Bioactive Products. (2014). The Polish pharmacopoeia. Pharmacopoea polonica. Vol. 1. Lumina the Publishing-Group.

19. European Communities. (1999). Directive 1999/2/EC of the European Parliament and of the Council of 22 February 1999 on the approximation of the laws of the Member States concerning foods and food ingredients treated with ionizing radiation. Official Journal of the European Communities, 13.3.1999, L66/16-L66/23.

20. European Communities. (1999). Directive 1999/3/ EC of the European Parliament and of the Council of 22 February 1999 on the establishment of a Community list of foods and food ingredients treated with ionizing radiation. Official Journal of the European Communities, 13.3.1999, L66/24-L66/25.

21. FAO/WHO. (2003). General standard for irradiated foods. Codex Stan 106-1983. Rev.1.

22. Ayscough, P. B. (1967). Electron spin resonance in chemistry. Leeds: Department of Physical Chemistry of Leeds University. 